\title{
Direct Measurement of Adhesion Energy of Monolayer Graphene As- Grown on Copper and Its Application to Renewable Transfer Process
}

Taeshik Yoon, $^{\dagger, \S}$ Woo Cheol Shin, ${ }^{\ddagger, \S}$ Taek Yong Kim, ${ }^{\ddagger}$ Jeong Hun Mun, ${ }^{\ddagger}$ Taek-Soo Kim, ${ }^{*} \dagger$ and Byung Jin Cho*,

${ }^{\dagger}$ Department of Mechanical Engineering and ${ }^{\ddagger}$ Department of Electrical Engineering, KAIST, Daejeon 305-701, Korea

Supporting Information

ABSTRACT: Direct measurement of the adhesion energy of monolayer graphene as-grown on metal substrates is important to better understand its bonding mechanism and control the mechanical release of the graphene from the substrates, but it has not been reported yet. We report the adhesion energy of large-area monolayer graphene synthesized on copper measured by double cantilever beam fracture mechanics testing. The adhesion energy of $0.72 \pm 0.07 \mathrm{~J} \mathrm{~m}^{-2}$ was found. Knowing the directly measured value, we further demonstrate the etching-free renewable transfer process of monolayer graphene that utilizes the repetition of the mechanical delamination followed by the regrowth of monolayer graphene on a copper substrate.

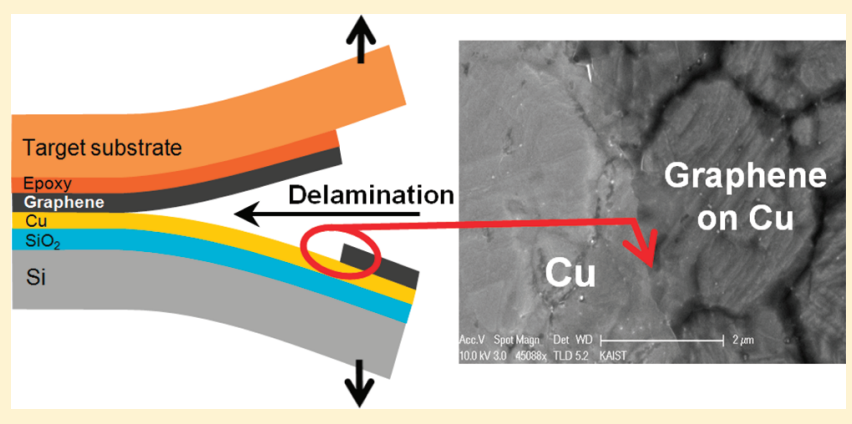

KEYWORDS: Graphene, adhesion, delamination, transfer, flexible electronics

$\mathrm{D}$ espite the intense interest in the measurements and applications of exceptional physical properties of graphene, ${ }^{1-11}$ the adhesion energy of monolayer graphene asgrown on metal substrates has not been reported thus far, although the adhesion energy of graphene flakes already transferred to other substrates has been reported. ${ }^{12-14}$ However, the adhesion energy of as-grown graphene on metal substrates should be measured not only for scientific interest in a better understanding of its bonding mechanism, but also for a technological breakthrough that would enable the precise control of the mechanical release of large-area graphene from metal substrates without the usage of existing wet etching methods. ${ }^{10,15-21}$

Large-area and high-quality graphene synthesized on metal substrates currently requires wet etching of the metal substrates to transfer the graphene to target substrates. ${ }^{10,15-21}$ However, the etching processes incur structural and chemical damage in the graphene. Moreover, it is time-consuming and inevitably requires the disposal of the metal substrates after only a single use, generating chemical waste, which significantly limits the cost-competitive and environmentally friendly mass production of graphene devices. Here, we developed an etching-free renewable transfer process of large-area monolayer graphene that utilizes the repetition of the mechanical delamination followed by the regrowth of monolayer graphene on a copper substrate. For precise control of the delamination process, the adhesion energy of the as-grown graphene on copper should be known, which we for the first time measured directly and accurately. After the transfer of the graphene layer, the bare copper substrate is subjected to the same graphene growth process and a new monolayer graphene is regrown on it. We further demonstrated that high-quality monolayer graphene can be repeatedly regrown after this etching-free mechanical transfer process without damaging the copper substrate. With the directly transferred graphene onto a flexible substrate, a topgate graphene field effect transistor (FET) with superior topgate modulation and bending stability was fabricated.

Graphene was synthesized on a $\mathrm{Cu} / \mathrm{SiO}_{2} / \mathrm{Si}$ wafer (see Supporting Information for graphene syntheses). The graphene was characterized by Raman spectroscopy and optical transmittance, confirming that high-quality monolayer graphene with a low defect density was synthesized on the copper substrate over a large area with excellent uniformity (Figures S1 and S2 in Supporting Information). The adhesion energy between the as-grown graphene and the copper substrate was measured by double cantilever beam (DCB) fracture mechanics testing $^{22-24}$ with a high-precision micromechanical test system (Delaminator Adhesion Test System; DTS Company, Menlo Park, CA). DCB specimens (inset of Figure 1) were fabricated by the epoxy bonding technique (see Supporting Information for the specimen preparation). Each specimen was loaded and unloaded under a constant displacement rate of $5 \mu \mathrm{m} \mathrm{s}^{-1}$ while the applied load was continuously monitored as a function of the displacement (Figure 1). It was observed that graphene delaminated from epoxy rather than from copper for displacement rates lower than $5 \mu \mathrm{m} \mathrm{s}^{-1}$. Further research is required

Received: November 23, 2011

Revised: January 11, 2012

Published: February 15, 2012 


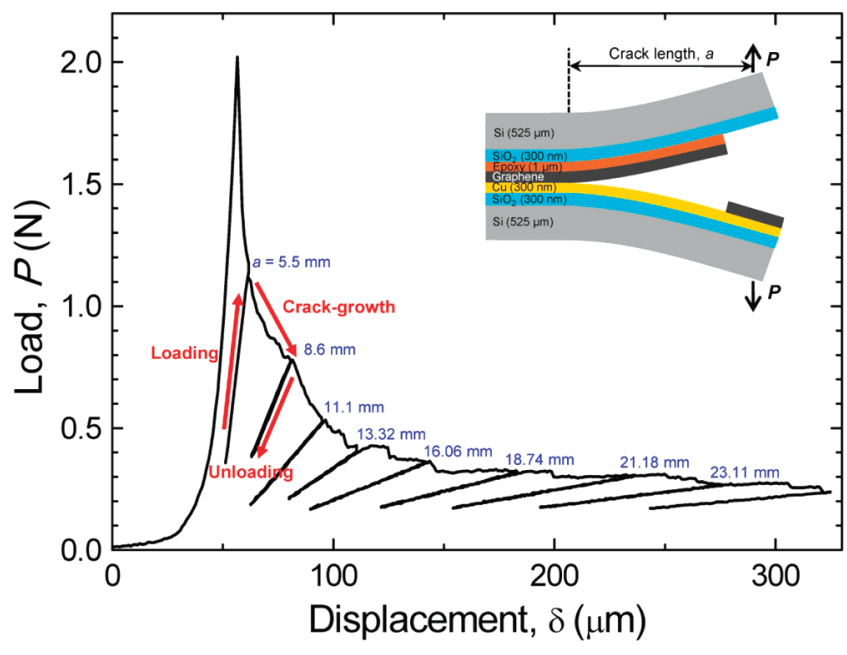

Figure 1. Measurement of the adhesion energy of graphene by DCB fracture mechanics testing. A schematic of the DCB specimen is shown in the inset. For the test, both Si beams are loaded and unloaded at a constant displacement rate while the applied load is monitored as a function of the displacement. Multiple loading/crack-growth/unloading cycles were performed to measure the crack lengths and the adhesion energy of the as-grown graphene on copper. The measured crack length, $a$, for each cycle is shown. regarding the rate-dependent fracture. ${ }^{25,26}$ Initially, a specimen was loaded elastically, but once the strain energy release rate exceeded the adhesion energy of the graphene/copper interface, crack growth occurred within the interface. At this critical load, the load-versus-displacement curve starts to decrease, reflecting changes in specimen compliance with the extension of the crack. Following a several-millimeter crack extension, the beam was partially unloaded and then subjected to further multiple instances of loading/crack-growth/unloading cycles, as shown in Figure 1, in order to obtain multiple values of the adhesion energy using a compliance-based model $^{22}$ (see Supporting Information for DCB testing and data analysis).

The adhesion energy value of $0.72 \pm 0.07 \mathrm{~J} \mathrm{~m}^{-2}$ was measured in our research and it is close to other such values calculated through quantum simulations. ${ }^{27-29}$ However, it is $60 \%$ larger than the adhesion energy of $0.45 \pm 0.02 \mathrm{~J} \mathrm{~m}^{-2}$ for monolayer graphene already transferred onto a $\mathrm{SiO}_{2}$ substrate. ${ }^{14}$ This indicates that the interatomic force between graphene and metal is stronger than the typical van der Waals force between graphene and a dielectric material. We believe the stronger bonding is due to an increase in the electronic density at the interface between the graphene and the metal, although the bonding type is basically van der Waals adhesion.
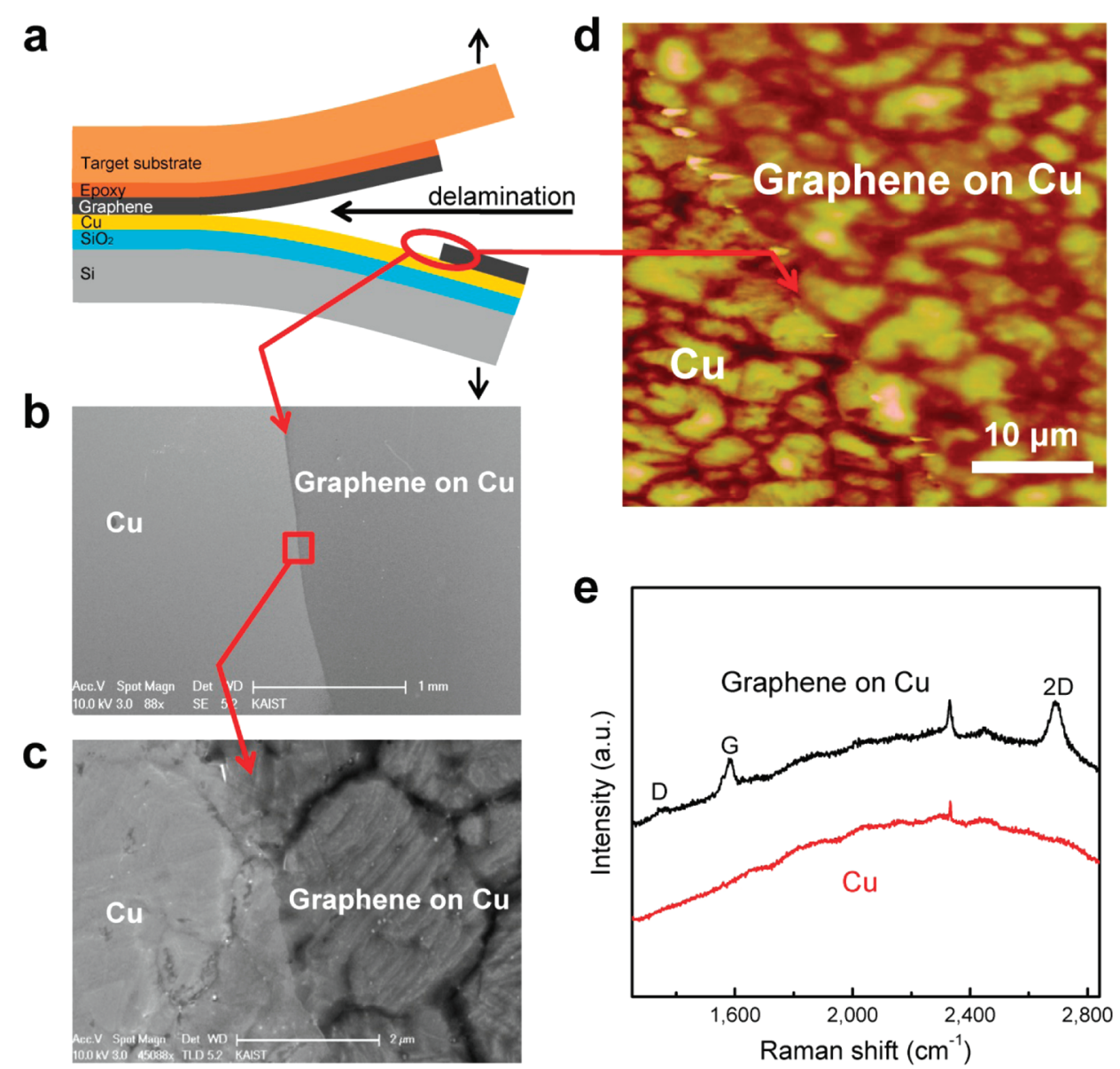

Figure 2. Etching-free selective mechanical transfer of large-area monolayer graphene. (a) Schematic of selective graphene transfer to a target substrate using the mechanical delamination process. (b) Low-magnification (the scale bar is $1 \mathrm{~mm}$ ) SEM image of a selectively graphenedelaminated copper surface. (c) High-magnification (the scale bar is $2 \mu \mathrm{m}$ ) SEM image. (d) AFM image of a selectively graphene-delaminated copper surface. (e) Raman spectra of the graphene-delaminated bare copper (the lower spectrum) and of the graphene-covered copper (the upper spectrum). 


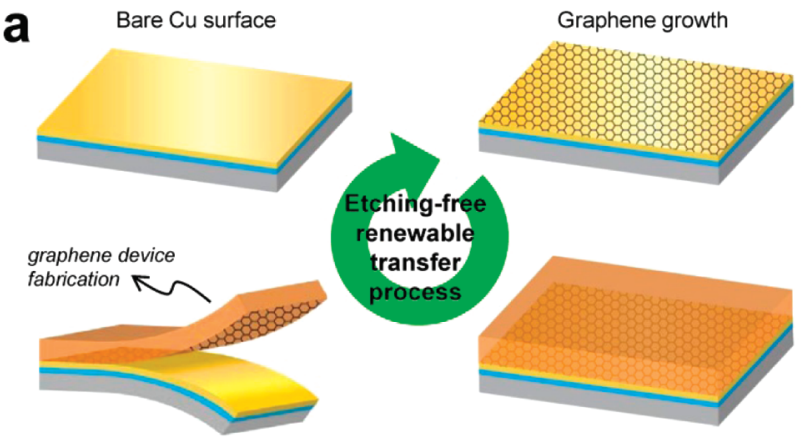

Graphene transfer

Bonding to a target substrate

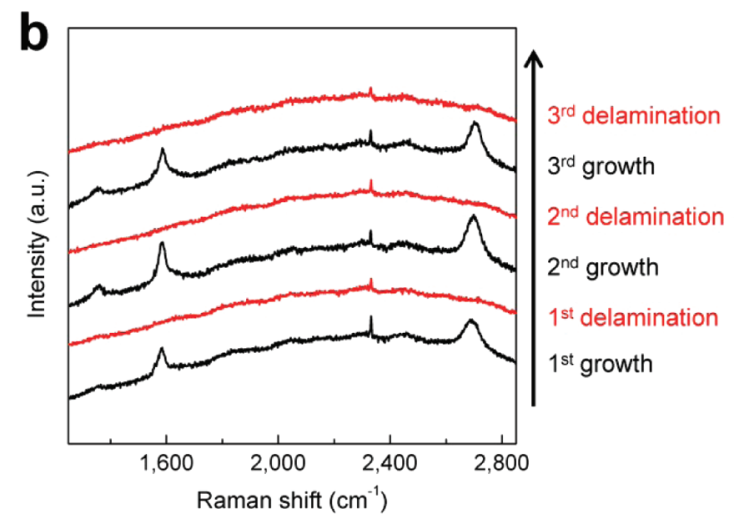

Figure 3. Etching-free renewable transfer process of large-area monolayer graphene. (a) Schematic of the steps of the etching-free renewable graphene transfer process. A target substrate is bonded to as-grown graphene on copper and the graphene is then transferred to the target substrate by mechanical delamination. The graphene-delaminated bare copper is employed again for the further graphene regrowth process. (b) Raman spectra of graphene-delaminated bare copper and synthesized graphene on copper. The initial as-grown graphene on copper (first growth) is transferred to a target substrate and graphene-delaminated bare copper is obtained (first delamination). Graphene is regrown on the bare copper (second growth). These procedures are repeated multiple times to demonstrate that the etching-free transfer process is renewable.

To the best of our knowledge, this has not been explicitly demonstrated elsewhere and therefore requires further study.

The direct measurement of the adhesion energy is critically required to develop a repeatable and reproducible mechanical transfer process of monolayer graphene as grown on copper. Without knowing the adhesion energy, it is hard to even guess how much force is needed to exfoliate the graphene, which has rendered the existing mechanical exfoliation process highly empirical. Consequently the wet chemical etching process is rather predominantly used for the release of large-area graphene synthesized on a metal substrate despite its disadvantages such as the generation of graphene damage and chemical waste, and the disposal of the metal substrates after only a single use. However, knowing the accurately measured adhesion energy, we were able to determine what kind of adhesive to use and how much force to apply in order to precisely overcome the adhesion between graphene and a copper substrate.

Knowing the accurately measured adhesion energy of the asgrown graphene on the copper substrate, we demonstrate that a selected area of the graphene can be picked up and transferred directly from the copper substrate to a target substrate without any etching process. Graphene on copper was selectively bonded to a target flexible polyimide (PI) substrate with the same epoxy used for the DCB specimen and mechanical loading was applied at the end of the specimen to delaminate the graphene from the copper substrate (Figure 2a). Scanning electron microscope (SEM) images show that etching-free mechanical delamination was achieved over a large area (Figure 2b) with submicrometer high precision (Figure 2c). The borderline between the graphene-delaminated bare copper and the graphene-covered copper is clearly seen. The mechanical delamination was also confirmed by atomic force microscopy (AFM), as shown in Figure 2d, which exhibits a rougher surface of the graphene-delaminated bare copper, in clear contrast with the smoother surface of the graphene-covered copper. Figure $2 \mathrm{e}$ shows the Raman spectra ${ }^{30}$ of the graphene-delaminated bare copper (the lower spectrum) and the graphene-covered copper (the upper spectrum). The Raman spectrum of the graphene-delaminated bare copper shows no graphene peaks, thus indicating that the graphene is completely released from the copper surface, while the peaks for the graphene on the copper are identical to those of the as-grown graphene (The right inset of Figure S1 in Supporting Information).

The most important advantage of the etching-free mechanical transfer process is that the copper substrate is intact, as a copper etchant is not used. Therefore, the substrate is reusable for further multiple graphene growth and transfer processes. Thus far, wet chemical etching has been considered to be an inevitable process to release as-grown graphene from metal substrates. However, the time-consuming etching process is associated with the generation of hazardous chemical waste, ${ }^{31}$ and the resulting copper solution is a serious source of water pollution. Graphene tends to deteriorate when using this process as well. Most importantly, the metal layer is destroyed by the etching process after a single-time graphene transfer. Therefore, a graphene transfer process involving wet chemical etching is not suitable for the cost-competitive and environmentally friendly mass production of graphene devices

Here, we demonstrate an etching-free renewable transfer process of monolayer graphene involving the repetitive etchingfree mechanical delamination followed by regrowth of a monolayer graphene on a copper substrate (Figure 3a). A 50 $\mu \mathrm{m}$ thick PI film as a target substrate is bonded to the as-grown graphene with epoxy. After the mechanical transfer of the graphene layer to the target substrate, which can be directly used for the fabrication of graphene devices, the graphenedelaminated bare copper substrate is subjected to the initial graphene growth step and a new monolayer of graphene is regrown on it. This etching-free renewable transfer process can be repeated multiple times without the copper substrate being lost or damaged. Figure $3 \mathrm{~b}$ shows the Raman spectra of the synthesized graphene on copper and of the graphenedelaminated bare copper during the renewable transfer process. The initial graphene is synthesized on a copper substrate, as confirmed by the Raman spectrum (first growth in Figure $3 b$ ), showing the high quality of the graphene. After graphene delamination, a bare copper surface is obtained (first delamination in Figure 3b) and is reused for another instance of graphene synthesis. Multiple regrowth/transfer cycles of the graphene are achieved without damaging the copper substrate and the graphene, as shown in Figure $3 \mathrm{~b}$.

With the graphene directly transferred to a flexible PI substrate, we demonstrate that simple yet effective fabrication 
a
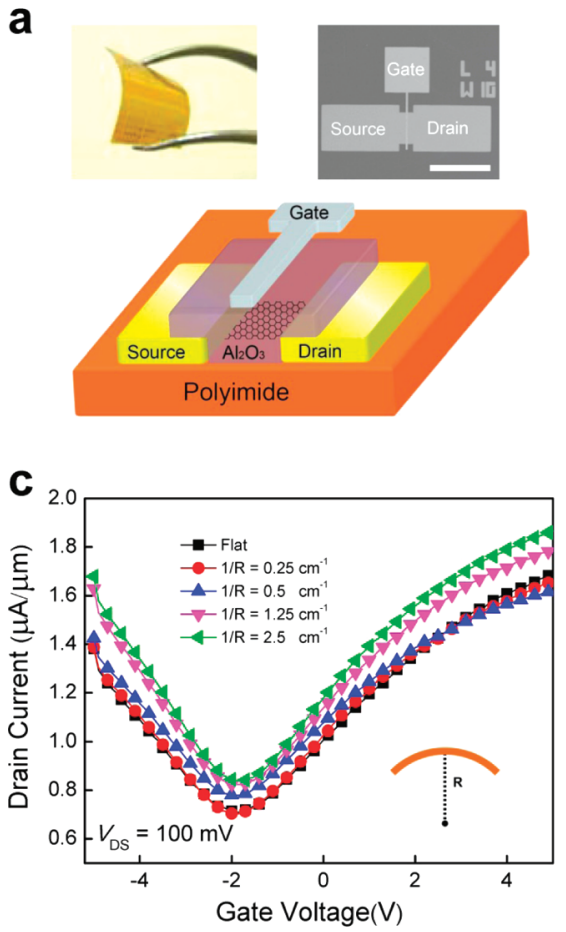

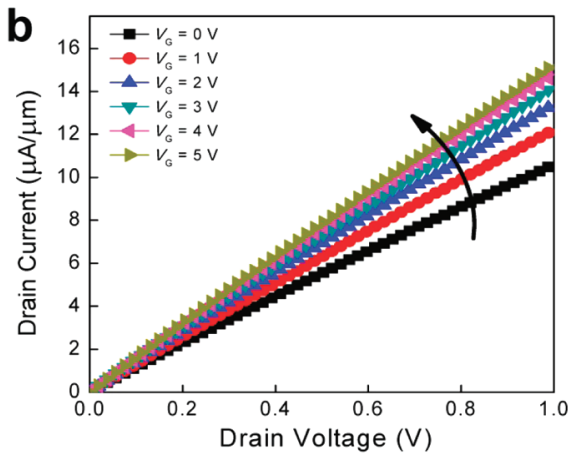

d

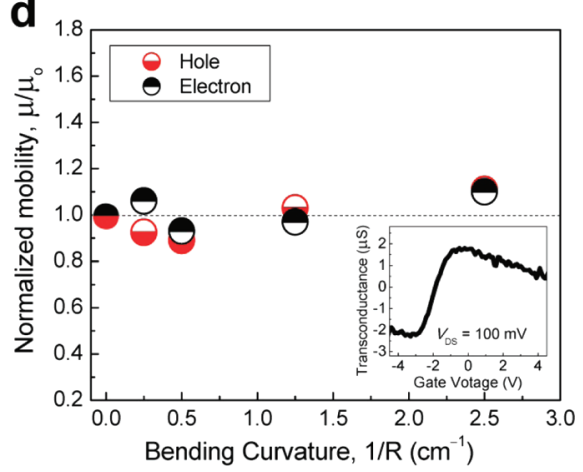

Figure 4. Demonstration of flexible top-gate graphene FET on a polyimide substrate. (a) The top left is an optical image of the flexible top-gate graphene FETs on a polyimide substrate and the top right is a SEM image of the FET (the scale bar is $200 \mu \mathrm{m}$ ). The bottom is a schematic of the FET. (b) Output characteristics $\left(I_{\mathrm{DS}}-V_{\mathrm{DS}}\right)$ of the graphene FET at various gate voltages. The gate modulation of the graphene channel conductance is evident. (c) Transfer characteristics $\left(I_{\mathrm{DS}}-V_{\mathrm{GS}}\right)$ of the graphene FET for different bending curvatures. The bended devices exhibit no change in the Dirac voltage. (d) Normalized hole and electron mobility as a function of the bending curvature. The mobility change is within $10 \%$.

of flexible graphene devices can be achieved by utilizing the proposed etching-free transfer process. To date, the fabrication of graphene-based FET has been carried out using a wet transfer process including metal etching which requires unwanted steps such as additional annealing. . $^{17,21}$ These steps have unfavorable effects on graphene, ultimately leading to unreliable device characteristics. In our research top-gate graphene FET was fabricated by means of the direct transfer of graphene to a flexible PI substrate without the undesirable copper etching process (see Supporting Information for the fabrication details). The fabricated top-gate graphene FET is shown in Figure 4a. Figure $4 \mathrm{~b}$ shows the output characteristic $\left(I_{\mathrm{DS}}-V_{\mathrm{DS}}\right)$ of the top-gate graphene $\mathrm{FET}$ at various gate voltages. The graphene FET fabricated based on the single step transfer process exhibits good gate modulation of the channel conductivity. The drain current shows almost linear dependence with respect to the drain-source voltage, which is a typical characteristic of graphene FET due to the zero-bandgap of graphene. ${ }^{1,2,4}$ Figure $4 \mathrm{c}$ shows the transfer characteristics $\left(I_{\mathrm{DS}}-\right.$ $V_{\mathrm{G}}$ ) with various bending curvatures of the substrate. The topgate graphene FET on the PI substrate exhibits excellent flexibility and no change in charge neutral point, Dirac voltage $\left(V_{\text {Dirac }}\right)$, up to $2.5 \mathrm{~cm}^{-1}$ of bending curvature. A stable $V_{\text {Dirac }}$ value after bending is desirable for flexible device applications, because it determines important device parameters such as the drain current level and the conduction carrier type. From the peak transconductance values (inset of Figure $4 \mathrm{~d}$ ) the field effect mobility, ${ }^{11} \mu=L g_{\mathrm{m}} / W V_{\mathrm{ds}} C_{\mathrm{ox}}$, was extracted for different bending curvatures (Figure $4 \mathrm{~d}$ ). The mobility change after the bending of the device was within $10 \%$.

In conclusion, we have for the first time directly measured the adhesion energy of large-area monolayer graphene as-grown on a copper substrate by DCB fracture mechanics testing. We have further demonstrated the etching-free renewable transfer process of the graphene by utilizing the repetition of the mechanical delamination followed by the regrowth of monolayer graphene on a copper substrate. This result can be used to enable the cost-competitive and environmentally friendly mass production of graphene devices.

\section{ASSOCIATED CONTENT}

\section{Supporting Information}

Additional information regarding (1) syntheses and characterizations of graphene, (2) DCB specimen preparation, testing and data analysis, and (3) FET fabrication. This material is available free of charge via the Internet at http://pubs.acs.org.

\section{AUTHOR INFORMATION}

\section{Corresponding Author}

*E-mail: (T.-S.K.) tskim1@kaist.ac.kr; (B.J.C.) bjcho@ee.kaist. ac.kr.

\section{Author Contributions}

${ }^{\S}$ T. S. Yoon and W. C. Shin contributed equally to this work. Notes

The authors declare no competing financial interest.

\section{ACKNOWLEDGMENTS}

This work was supported by research grants from the National Research Foundation of Korea (NRF) (Grants 2011-0019177 and 2011-0029835), the MCP Core Technologies for the Next Generation Project of the Ministry of Knowledge Economy of Korea and the Center for Advanced Soft Electronics (Grants 
2011-0031638) under the Global Frontier Research Program of the Ministry of Education, Science and Technology, Korea .

\section{REFERENCES}

(1) Novoselov, K.; Geim, A.; Morozov, S.; Jiang, D.; Zhang, Y.; Dubonos, S.; Grigorieva, I.; Firsov, A. Science 2004, 306 (5696), 666.

(2) Novoselov, K.; Geim, A.; Morozov, S.; Jiang, D.; Katsnelson, M.; Grigorieva, I.; Dubonos, S.; Firsov, A. Nature 2005, 438, 197.

(3) Frank, I.; Tanenbaum, D.; Van Der Zande, A.; McEuen, P. J. Vac. Sci. Technol., B 2007, 25, 2558.

(4) Geim, A. K.; Novoselov, K. S. Nat. Mater. 2007, 6 (3), 183-191.

(5) Bunch, J. S.; Verbridge, S. S.; Alden, J. S.; Van Der Zande, A. M.; Parpia, J. M.; Craighead, H. G.; McEuen, P. L. Nano Lett. 2008, 8 (8), $2458-2462$.

(6) Lee, C.; Wei, X.; Kysar, J. W.; Hone, J. Science 2008, 321 (5887), 385.

(7) Nair, R.; Blake, P.; Grigorenko, A.; Novoselov, K.; Booth, T.; Stauber, T.; Peres, N.; Geim, A. Science 2008, 320 (5881), 1308.

(8) Castro Neto, A.; Guinea, F.; Peres, N.; Novoselov, K.; Geim, A. Rev. Mod. Phys. 2009, 81, 109-162.

(9) Stankovich, S.; Dikin, D. A.; Dommett, G. H. B.; Kohlhaas, K. M.; Zimney, E. J.; Stach, E. A.; Piner, R. D.; Nguyen, S. B. T.; Ruoff, R. S. Nature 2006, 442 (7100), 282-286.

(10) Bae, S.; Kim, H.; Lee, Y.; Xu, X.; Park, J. S.; Zheng, Y.; Balakrishnan, J.; Lei, T.; Kim, H. R.; Song, Y. I. Nat. Nanotechnol. 2010, 5 (8), 574-578.

(11) Schwierz, F. Nat. Nanotechnol. 2010, 5 (7), 487-496.

(12) Sen, D.; Novoselov, K. S.; Reis, P. M.; Buehler, M. J. Small 2010, 6 (10), 1108-1116.

(13) Zong, Z.; Chen, C. L.; Dokmeci, M. R.; Wan, K. J. Appl. Phys. 2010, 107 (2), 026104-026104-3.

(14) Koenig, S. P.; Boddeti, N. G.; Dunn, M. L.; Bunch, J. S. Nat. Nanotechnol. 2011, 6 (9), 543-546.

(15) Reina, A.; Jia, X.; Ho, J.; Nezich, D.; Son, H.; Bulovic, V.; Dresselhaus, M. S.; Kong, J. Nano Lett. 2008, 9 (1), 30-35.

(16) De Arco, L. G.; Zhang, Y.; Kumar, A.; Zhou, C. IEEE Trans. Nanotechnol. 2009, 8 (2), 135-138.

(17) Kim, K. S.; Zhao, Y.; Jang, H.; Lee, S. Y.; Kim, J. M.; Ahn, J. H.; Kim, P.; Choi, J. Y.; Hong, B. H. Nature 2009, 457 (7230), 706-710.

(18) Levendorf, M. P.; Ruiz-Vargas, C. S.; Garg, S.; Park, J. Nano Lett. 2009, 9 (12), 4479-4483.

(19) Li, X.; Cai, W.; An, J.; Kim, S.; Nah, J.; Yang, D.; Piner, R.; Velamakanni, A.; Jung, I.; Tutuc, E. Science 2009, 324 (5932), 1312.

(20) Li, X.; Zhu, Y.; Cai, W.; Borysiak, M.; Han, B.; Chen, D.; Piner,

R. D.; Colombo, L.; Ruoff, R. S. Nano Lett. 2009, 9 (12), 4359-4363.

(21) Lee, Y.; Bae, S.; Jang, H.; Jang, S.; Zhu, S. E.; Sim, S. H.; Song,

Y. I.; Hong, B. H.; Ahn, J. H. Nano Lett. 2010, 10 (2), 490-493.

(22) Kanninen, M. F. Int. J. Fract. 1973, 9 (1), 83-92.

(23) Hohlfelder, R. J.; Maidenberg, D. A.; Dauskardt, R. H.; Wei, Y.; Hutchinson, J. W. J. Mater. Res. 2001, 16 (1), 243-255.

(24) Kim, T.; Tsuji, N.; Matsushita, K.; Kobayashi, N.; Chumakov, D.; Geisler, H.; Zschech, E.; Dauskardt, R. H. J. Appl. Phys. 2008, 104, 074113.

(25) Chaudhury, M. K. J. Phys. Chem. B 1999, 103 (31), 6562-6566.

(26) Meitl, M. A.; Zhu, Z. T.; Kumar, V.; Lee, K. J.; Feng, X.; Huang, Y. Y.; Adesida, I.; Nuzzo, R. G.; Rogers, J. A. Nat. Mater. 2005, 5 (1), 33-38.

(27) Lahiri, J. New J. Phys. 2011, 13, 025001.

(28) Xu, Z.; Buehler, M. J. J. Physics: Condens. Matter 2010, 22, 485301.

(29) Vanin, M.; Mortensen, J. J.; Kelkkanen, A.; Garcia-Lastra, J. M.; Thygesen, K. S.; Jacobsen, K. W. Phys. Rev. B 2010, 81 (8), 081408.

(30) Ferrari, A.; Meyer, J.; Scardaci, V.; Casiraghi, C.; Lazzeri, M.; Mauri, F.; Piscanec, S.; Jiang, D.; Novoselov, K.; Roth, S. Phys. Rev. Lett. 2006, 97 (18), 187401.

(31) Gilles, D. G. J. Environ. Eng. 1994, 120, 72. 